\title{
Une histoire de chair et de fureur : étude de la sexualité dans Kamouraska
}

ADRIEN RANNAUD

CRILCQ - UNIVERSITÉ LAVAL

Résumé : Cet article propose d'aborder le déploiement et les conséquences de la sexualité dans Kamouraska d'Anne Hébert. À partir du personnage d'Elisabeth, héroïne en proie à une confusion des sentiments et des désirs, nous postulons que le système agentif est basé sur l'économie sexuelle des personnages féminins, et que les rapports sexuels dans le roman constituent un champ de bataille réinvestissant les enjeux idéologiques du $19^{e}$ siècle québécois.

Mots-clés : Anne Hébert, Kamouraska, Sexualité, Système agentif, Assujettissement.

En 1970, le journaliste et écrivain Jean Éthier-Blais écrivait à propos de Kamouraska : « [c']est un livre de sexualité lourde et un peu écœurante. » (Éthier-Blais, 1970 : 12) De l'autre côté du spectre critique, Ivanhoé Beaulieu qualifiait I'œuvre de roman d'amour à « [I]'allure fleur bleue ». (Beaulieu, $1970: 50$ ) Ces deux prises de position, aussi différentes soient-elles, mettent de l'avant l'incroyable palette discursive développée par la critique pour décrire l'exacerbation des passions dans le roman d'Anne Hébert. Si Kamouraska impose une rupture dans la production littéraire de son auteure, à en croire la critique de l'époque ${ }^{1}$, $\mathrm{c}^{\prime}$ est entre autres par son exploration des « arcanes de la passion amoureuse » (Ancrenat et Marcheix dans Fuvres complètes II, 2013 : 184), révélant conjointement les élans sublimes et les affres triviales d'un personnage désirant, Elisabeth d'Aulnières. Dans le sillage des propos des deux commentateurs, I'appréciation publique cristallise son discours autour de I'héroïne du roman $\left(O C I I^{2}: 184\right)$. Tantôt héritière des grands drames shakespeariens (Jardin, 1970 : 25), tantôt « Desqueyroux aux cuisses ruisselantes » (Vigneaux, 1971 : 101), Elisabeth, narratrice et personnage central du roman, est cette femme

1. Sur la réception critique de Kamouraska, on lira notamment la présentation du roman par Anne Ancrenat et Daniel Marcheix, et plus précisément la section « Réception critique » (Fuvres complètes II, 2013 : 181-186).

2. OCII pour Fuvres complètes II, 2013. 
troublée et troublante, une « ardente femelle » (Mattéi, 1970) dont les pulsions et les sentiments semblent actionner et régir l'intrigue et les différents plans du récit, que ce soit à Québec dans les années 1860, ou à Sorel et à Kamouraska lors des Rébellions.

Cet aperçu de la réception critique souligne l'évidence de la lecture de la sexualité dans Kamouraska, ainsi que la gêne qui l'accompagne. L'œuvre est empreinte du désir charnel. Et l'amour, pour peu qu'il soit représenté dans le roman, n'apparaît en premier lieu que par le prisme d'une attirance sexuelle entre I'homme et la femme. Aussi, nous essaierons, dans cet article, de montrer comment la sexualité est à la fois un tabou, mais aussi la pierre angulaire de la socialité du roman d'Anne Hébert. Comment la sexualité influence-t-elle les liens entre I'héroïne et les autres personnages ? Au sein d'un espace canadien-français janséniste bouleversé par les insurrections, quel regard Hébert porte-t-elle sur l'économie des désirs de l'époque?

Dans la foulée du commentaire d'Éthier-Blais évoqué précédemment, nous faisons le pari d'étudier le déploiement de la sexualité dans les recoins du tissu romanesque. En nous attachant au parcours diégétique de l'héroïne, force sera de constater que les nombreuses confusions d'Elisabeth entre sentiment amoureux et plaisir de la chair contaminent le système agentif ${ }^{3}$. À l'aide des principes énoncés par Nathalie Heinich sur les « états de femme » et les modulations de la disponibilité sexuelle dont ils rendent compte (Heinich, 1996 et 2003), nous déborderons de la stricte analyse d'Elisabeth pour nous pencher sur les autres personnages féminins et leur rapport au sexuel. Nous verrons ainsi comment la sexualité et le système agentif dans Kamouraska sont intrinsèquement liés, et de quelle manière les désirs des personnages sont investis à la fois comme un champ de bataille idéologique, mais aussi comme le lieu d'une révolte du sujet, féminin de surcroît.

Du roman d'amour au roman de la chair

En 1931, scandalisé par le roman La chair décevante (1931) de Jovette Bernier, Camille Roy écrivait une phrase restée célèbre :

3. Nous entendons par « système agentif » I'ensemble des puissances d'agir des personnages, puissances structurées et structurantes par leur mise en relation et par les conséquences des actions de chacun. Nous nous inspirons ici des travaux sur la notion d'agency, notamment ceux de Barbara Havercroft (1999). L'agency (que nous traduirons par « agentivité » et par «puissance d'agir » dans le texte) est «la capacité d'agir dans sa vie, de réaliser son potentiel » (Havercroft, $1999: 94$ ), mais aussi la force d'action d'un personnage dans le récit. Ici, le système agentif est organisé, comme on le verra, autour de la puissance d'agir d'Elisabeth. 
Il y a une différence entre un roman d'amour et un roman de la chair. Tous deux sont d'ordinaire étroitement apparentés. Mais il y a, d'ordinaire, ou il peut y avoir parfois, dans le premier, une certaine noblesse de sentiment, un idéalisme généreux, que I'on ne trouve pas dans le second. Celui-ci nous montre ce qu'il y a de plus inférieur dans la passion humaine, et ce qu'elle a de plus égoïste, l'instinct physique de la chair décevante. (Roy, 1931: 97)

On ne s'y trompera pas, ni le roman d'amour, très en vogue durant l'entre-deuxguerres, ni « le roman de la chair » ne trouvent grâce auprès de l'homme de lettres. En ce qui concerne le second type de roman, le plus condamnable selon Roy, lisons derrière les mots : le « roman de la chair », c'est le roman de la sensualité, de l'incarnation des passions humaines, de la corporalité du sujet. Et derrière la sensualité, toute une langueur d'ordre sexuel.

De La chair décevante à Kamouraska, une quarantaine d'années et de multiples changements, tant dans le champ littéraire que dans l'espace canadien-français devenu québécois, ont ouvert la voie à de nouvelles façons d'écrire et de lire, ainsi qu'à de nouveaux thèmes romanesques. Mais la distinction persiste entre le sentiment noble qu'est l'amour, et la chair incohérente et dangereuse dans son incandescence. En témoigne un article d'Albert Le Grand publié en 1971, qu'est une des premières analyses du roman de Hébert. L'article de Le Grand questionne la valeur du fait divers, et plus encore, l'ambivalence d'un récit oscillant entre mensonge et vérité, entre duplicité des masques et souci d'un absolu sans contrainte pour Elisabeth. D'une part, l'auteur suggère que le sentiment amoureux, et plus généralement la Fable (Le Grand, 1971 : 132) - du latin fabula, le mensonge - s'annulent d'eux-mêmes dans Kamouraska : I'amour pour George Nelson n'aurait « jamais vraiment existé, même si Elisabeth voudrait se persuader du contraire. » (Le Grand, 1971 : 140) D'autre part, le critique relève le combat permanent qui semble être mené contre «l'instinct de la chair devenu l'instinct du mal. » (Le Grand, 1971 : 133) On reconnaît là un certain manichéisme janséniste qui régit le roman hébertien. D'un côté se trouve l'amour factice servant à exalter le Bien ; de l'autre côté, c'est la chair maléfique qui détourne des devoirs chrétiens et du bien commun de la société.

Le Grand attire notre attention sur ce qu'on pourrait appeler, à la suite de Stefan Zweig, La confusion des sentiments (1927). En effet, durant la majeure partie du roman, I'héroïne croit agir au nom de l'amour ; mais c'est le désir sexuel qui préside à la trajectoire romanesque d'Elisabeth. Très tôt, la jeune fille est attirée par les hommes. Alors qu'elle vient d'être tondue, privée d'un signe social de sa féminité 
en construction, Elisabeth se prend pour un garçon et rejoint « tout un tas de petits gamins » (Kamouraska : 53) pour pêcher la barbote. Il n'y a a priori rien de sexuel dans ce souvenir. Néanmoins, il est le premier instant d'une rencontre avec le sexe masculin. Une rencontre marquante qui plus est, puisque plus tard, elle souhaitera renouer avec cet événement de l'enfance : « Je voudrais bien sortir moi aussi. Aller pêcher la barbote comme quand j'étais petite ! Avec des garçons ! ( $\left.K^{4}: 59\right)$. Ce n'est pas la pêche qui intéresse Elisabeth, mais les rencontres qu'elle y ferait.

Rappelons que I'héroïne est orpheline de père et vit entourée de sa mère et de ses tantes, les trois sœurs Lanouette. Elle reçoit de ces dernières une éducation stricte : elle est confinée dans un environnement féminin, et on lui interdit de rejoindre les garçons. Les Lanouette ne manquent pas de lui faire la leçon en matière de politesse, de religion ou de langue anglaise. Or, lorsqu'il s'agit d'expliquer ses premières règles à la jeune fille, les réponses sont sibyllines : « - Vous êtes sûre, tante Angélique, que cela va recommencer comme ça tous les mois ? - Oui, ma chérie. Nous y passons toutes. C'est la loi du monde. » $(K: 55)$ Vieilles filles de leur condition, les tantes restent «pudique[s] » et « confuse[s] » $(K: 55)$ devant les menstruations. Aussi l'expérience de la sexualité chez Elisabeth commence-t-elle par une incompréhension profonde des rapports entre les hommes et les femmes. L'absence de la mère qui pourrait faire l'éducation sexuelle de sa fille, et la mystification de « la loi du monde » par tante Angélique laissent Elisabeth dans une certaine ignorance à cet égard. Même Aurélie, qui « en sait autant sur la vie que les morts eux-mêmes » $(K: 59)$ refuse de répondre aux questions d'Elisabeth.

Cependant, en même temps que les tabous dominent la maison familiale de Sorel, la jeune fille devient adolescente et fait face à des sensations nouvelles. Elle interroge sa servante : «Et les garçons, Aurélie ? Parle-moi des garçons ? » ( $K: 63$ ) Mais il lui faut attendre les rencontres officielles avec les hommes dans le cadre du bal du gouverneur. Véritable entrée dans le monde, ce fameux passage obligé pour toute fille de bonne famille ${ }^{5}$, envisagé dans une logique plus marchande que romantique, le bal du gouverneur est aussi le moment d'une rencontre sensuelle avec les « garçons ». Elisabeth prend conscience des effets de son charme sur les hommes, notamment sur le Gouverneur qui lorgne le décolleté de la débutante tout en lui « respir[ant] dans le cou ». ( $K: 64)$ La jeune femme fait l'expérience du fantasme sexuel avec le

4. K pour Kamouraska, 1970.

5. «Moment où la fille nubile va se trouver publiquement exposée en tant que "fille à prendre", fiancée potentielle, officiellement candidate à la désirabilité et à son institutionnalisation par le mariage. » (Heinich, 1996 : 46) 
notable : il a quarante ans, il est un homme, contrairement aux « garçons endimanchés » $(K: 64)$ encore dans l'enfance. Il n'a rien du prince charmant, à en juger par «ses favoris rouillés » et son visage « tout rouge. » $(K: 64)$ Néanmoins, Elisabeth voit en lui un partenaire de danse expérimenté. Portée par la musique qui lui «monte à la tête » $(K: 64)$ et par l'honneur qui lui est réservé, elle envisage un instant l'idée d'un mariage avec le Gouverneur selon un enchâssement de phrases éloquent : «Ma mère dit encore qu'il faut me marier. Le Gouverneur a bien quarante ans, I'âge intéressant. » $(K: 64-65)$

La rencontre avec Antoine Tassy, cet homme « sensuel et viveur » (Smith, 19801981 : 70), ne fait qu'augmenter la méprise du personnage féminin au sujet de ses émotions. Au cours d'une partie de chasse, les deux jeunes gens se désirent du coin de I'œil : «Tu me devines, Antoine Tassy, et tu me traques [...]. Et moi aussi je te flaire et je te découvre. » $(K: 67)$ La jeune fille formule un fantasme, celui d'être couchée par Antoine «dans les joncs et la boue. » ( $K: 67)$ Elisabeth désire être salie et violentée, puisqu'elle se débat, en imagination, sous Antoine. Dans l'esprit de la jeune fille inexpérimentée, l'acte sexuel est à la fois une perdition et un plaisir espéré. Si la rencontre agit comme un coup de foudre, la narratrice se passe bien d'en utiliser les figures discursives, préférant de loin voir la parade amoureuse comme une chasse entre prédateur et proie... Une fois la demande en mariage faite par Antoine, elle s'interroge, faute d'obtenir des réponses du gynécée familial. Dupée, Elisabeth constate une antithèse entre « l'amour, la belle amour des chansons et des romans » $(K: 69)$ et les mystères de la « nuit de [I]a chair. » $(K: 69)$ Une première critique apparaît, celle de l'éducation des filles, à qui I'on fait croire à l'idée d'amour détaché de toute réalité matérielle. Elisabeth, (et derrière elle l'auteure Anne Hébert), questionne les faux semblants et l'imposture de son éducation : « Je te cache la vie et la mort derrière de grands paravents, brodés de roses et d'oiseaux exotiques. » $(K: 69)$

Le premier mariage d'Elisabeth révèle à cette dernière la tromperie dont elle a été victime, en même temps qu'elle est satisfaite dans son appétit sexuel. Elle se rend compte dès le jour des noces qu'elle a épousé « un homme qu['elle] n'aime pas » ( $K: 70)$ mais joue tout de même à la jeune épouse parfaite, telle une image d'Épinal. Tout en faisant route vers le domaine de son mari, elle est partagée entre le regret d'être mariée à Antoine et ce désir en elle, « le centre de [s]a vie ». ( $K: 71)$ C'est I'aveu d'un attrait pour la chair qu'elle partage avec son mari, dans une « connivence parfaite ». ( $K: 71-72)$ Néanmoins, l'expérience sexuelle du premier mariage d'Elisabeth reste difficile. Le premier coït est suggéré au lecteur par « [c]ette fraîche entaille 
entre ses cuisses ». ( $K: 73)$ Ce n'est ni la jouissance qui est exprimée, ni la fébrilité d'une première fois vécue, mais la blessure, la douleur d'un acte violent. Ensuite, I'héroïne subit son époux plus qu'elle ne l'aime, sauf dans les relations sexuelles où un partage ambigu se met en place. C'est le thanatos et l'éros, la promesse de la mort des deux époux complices par la corde du mariage ou le couteau, et le désir sexuel rédempteur, prêt à racheter les fautes du couple. Elisabeth semble fournir silencieusement cette explication à ses tantes :

De jour, je veux bien pleurer sur votre épaule. Voter la mort du coupable. N'être plus qu'un appât dolent aux yeux battus que I'on place en première ligne, afin de mieux confondre le mari monstrueux. [...] Mais de nuit, je redeviens la complice d'Antoine. Jusqu'au dégoût le plus profond. La terreur la plus folle. $(K: 99-100)$

« [L]'ivresse, le blasphème, la violence, l'amour et la dérision », « [d]e douleur ou de plaisir » $(K: 100)$ : le mariage fait peur à Elisabeth et en même temps la contente dans sa ferveur de la chair.

Déçue et excitée par un mari violent, la sexualité de l'héroïne se trouve exaltée par l'arrivée de George Nelson dans le paysage de Sorel. La première visite du docteur met en émoi Elisabeth dédoublée, celle qui se souvient et celle qui vit la visite médicale. La deuxième visite de George révèle une tension sexuelle naissante entre les deux personnages. Les «feu », « incendie » et « ravage » $(K: 112)$ réaffirment le topos occidental de l'amour qui embrase les cœurs. Mais la puissance de ce feu intérieur rappelle aussi la chair échauffée du personnage féminin. La rencontre avec George exacerbe les désirs d'Elisabeth qui voit enfin le plaisir charnel et l'amour réunis en une seule personne. Avec George, Elisabeth semble découvrir « ce désir absolu », qui mêle une passion dévorante et une sexualité enfin assumée. Dès lors, les vêtements tombent ( $K: 143$ ), les sexes se montrent et s'éveillent ( $K: 159)$, et la narratrice s'épanouit dans une jouissance qu'elle ne comprend pas (« Comment peuvent-ils désormais vivre comme tout le monde »[K:142]), et dont elle devient dépendante.

La suite, justement, s'enchaîne sur une incompréhension de la part des deux amants. Lorsqu'ils préparent le meurtre d'Antoine, Elisabeth et George agissent davantage dans le désir de tuer ce qui semble être un obstacle à leur bonheur que dans la volonté d'être réunis après le meurtre. Aucune prévision sur ce qu'il adviendrait par la suite : tout réside dans l'acte meurtrier. Une fois le mari assassiné, l'extase du complot passée, l'illusion s'estompe et la réalité des sens fait surface. Les deux 
amants se regardent et prennent conscience de l'erreur dans laquelle les a plongés leur désir : «Qui le premier de nous deux pleure doucement, la tête tombée sur sa poitrine ? Comme un pendu ? Qui ose répéter le mot "amour" et le mot "liberté", dans l'ombre, sans mourir de désespoir ? » ( $K: 241)$ Comme le pressentait Albert Le Grand, ce passage remet en question la nature des sentiments entre George et Elisabeth : était-ce vraiment de l'amour, ou une projection sentimentale entraînée par le désir sexuel entre les deux personnages ? Quoi qu'il en soit, les liens sont brisés entre les amants qui se maudiront mutuellement à la fin du roman. Quant au second mariage d'Elisabeth, cette dernière le confessait au début du roman : il est dénué d'amour, fait dans l'intérêt de l'honneur ( $K: 9)$.

Elisabeth se montre incapable de faire la différence entre ses illusions et la réalité libidinale. Perdue, I'héroïne se fie aux réflexes de sa chair qu'elle sublime dans le même temps. La situation s'inverse, et Elisabeth recherche le rapport sexuel comme condition d'accès à la passion amoureuse. Le schéma du roman sentimental conventionnel - où l'amour prime - est reconfiguré, laissant apparaître un tout autre développement romanesque : un personnage féminin fait l'apprentissage de son incarnation sensible. Or, Kamouraska n'est pas le roman «d'une » chair, mais bien de «la » chair dans son sens générique. La trame diégétique nous invite à suivre les réflexions et actions d'une héroïne troublée. Dès lors, prêter attention aux faits et gestes du personnage féminin principal revient aussi à questionner les rapports de ce dernier avec ses pairs ${ }^{6}$. On peut se demander comment la sexualité influence les rapports entre Elisabeth et les autres personnages féminins. En quoi l'économie sexuelle du roman nous renseigne-t-elle sur la puissance d'agir des femmes de Kamouraska?

États de femme et agentivités : la sexualité et le champ des possibles

Dans États de femme, Nathalie Heinich propose d'évaluer et de catégoriser les possibilités stratégiques des figures féminines dans les littératures occidentales. Deux critères guident l'analyse : d'une part, le statut économique du personnage féminin, d'autre part, sa disponibilité sexuelle. De la combinaison de ces deux critères émerge une typologie d'états tels que la «première » (femme mariée), la « seconde » (maîtresse, seconde femme) ou la «tierce » (gouvernante, veuve, vieille fille). En soulignant les «structures élémentaires de l'identité féminine »(Heinich, $1996: 327)$

6. «L'héroïne a souvent une sœur, une confidente, une amie proche ou lointaine qui lui sert de miroir, certes, mais dont elle est prête aussi à devenir le miroir. Tantôt l'autre femme sera très proche de l'héroïne, permettant alors l'expression d'un narcissisme fondamental, tantôt, au contraire, très différente, elle permettra, par sa différence même, d'exercer un étrange tropisme, de révéler à l'héroïne ce qu'elle n'est pas, ce qu'elle pourrait être, ce qu'elle aimerait être. » (Didier, 1991 : 27) 
dans la tradition littéraire, Heinich démontre que « par le type de rapport entretenu avec le monde viril, le pouvoir masculin [...] les femmes utilisent les hommes pour se démarquer les unes des autres, en fonction de leur relation à l'autre sexe ». (Heinich, 1996 : 336-337) Dans Les ambivalences de l'émancipation féminine, elle insiste sur la perception qu'a le personnage féminin de ses paires : une hiérarchie « entre elles » se construirait selon « un degré de légitimité du lien écono-micro-sexuel ». (Heinich, $2003: 12$ )

Les analyses de Heinich révèlent la construction de clichés féminins littéraires reposant directement sur une identification économico-sexuelle des femmes ${ }^{7}$. Dans Kamouraska, cette identité est instable, puisque le personnage principal et moteur de la narration change d'état de femme au fil des pages : jeune «fille à prendre », «première » officielle (mariage) et clandestine (adultère), veuve, à nouveau « première ». La classification des états de femme peut paraître réductrice. Mais elle permet de voir combien l'aspect sexuel déborde de l'imaginaire du personnage principal, dans un système narratif qui donne la parole à Elisabeth. Et si I'identité s'explique par la fonction économico-sexuelle, alors le système agentif en est foncièrement tributaire.

\section{Figure 1. Classification des états de femme dans le roman Kamouraska}

\begin{tabular}{|c|c|c|c|c|}
\hline & La première & & La tierce & \\
\hline 莣 & La première & $\begin{array}{c}\text { La veuve (ancienne } \\
\text { première) }\end{array}$ & La vieille fille & La servante \\
\hline 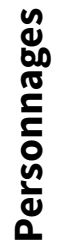 & Elisabeth & $\begin{array}{c}\text { Mme d’Aulnières } \\
\text { Caroline des } \\
\text { Rivières Tassy }\end{array}$ & $\begin{array}{l}\text { Les tantes Lanouette } \\
\text { Léontine Mélançon }\end{array}$ & $\begin{array}{l}\text { Aurélie } \\
\text { Florida }\end{array}$ \\
\hline
\end{tabular}

Elisabeth est le seul personnage féminin à bénéficier du statut privilégié de « la première ». Elle est la femme mariée, cet « état [...] que recherchent les filles autant que leur famille » (Heinich, $1996: 86$ ) ; elle a «l'honneur d'être Madame ». (K:53) C'est I'aboutissement heureux d'une jeune fille, celui dont on rêve dans les contes pour enfants ${ }^{8}$, un état qui donne accès à une place sociale de choix et à la sexualité tant attendue. Mais, ainsi que le rappelle Heinich, l'état de première est « loin d'être

7. De façon similaire, les travaux de Paola Tabet sur l'échange économico-sexuel dans les sociétés occidentales modernes traitent également de la valeur marchande de la sexualité féminine. On lira principalement La grande arnaque. Sexualité des femmes et échange économico-sexuel (2004).

8. Adrien Thério a livré une analyse intéressante de l'intertexte des contes merveilleux dans l'œuvre d'Anne Hébert. Selon Thério, dans Kamouraska, le mariage n'est bien sûr qu'un leurre, et le conte de fées se transforme en tragédie avant même que les époux ne soient partis pour le « royaume du prince » (Thério, 1971). 
monolithique ». (Heinich, $1996: 105)$ Il est menacé, soit par les autres (une autre femme, le mari lui-même), soit par les agissements de la première elle-même. Avec l'adultère, celle-ci deviendrait une « première clivée » entre mari et amant (Heinich, 1996 : 106). Dans Kamouraska, Elisabeth mène une double vie : un masque porté avec le mari et la société, une personnalité épanouie avec le docteur Nelson. De fait, la sexualité est elle-même clivée. Avec Antoine, I'acte sexuel est une « réconciliation » $(K: 146)$, un temps du consensus permettant le rétablissement des bonnes relations entre les deux époux pour sauver I'honneur familial. Avec George, les pulsions animales sous-tendent un plaisir qui dépasse les conventions sociales et révèle la femme derrière son état ( $K: 159)$.

Elisabeth est « chanceuse »: toutes les femmes du roman n'ont pas l'opportunité d'accéder à un état aussi prestigieux que celui de «première »; et le personnage principal observe ses paires en tenant compte de cette «infériorité ». Les autres personnages féminins relèvent d'une série d'états que Heinich regroupe sous le nom de « tierce $»^{9}$. C'est un personnage célibataire, ce qui s'explique par une profession, par une situation familiale ou par un choix délibéré. Dans la diégèse, elle n'entretient pas a priori de rapports amoureux ou sexuels avec un homme : elle est voyante, mais invisible ; en état de tiers, c'est-à-dire intégrée au système agentif, mais placée à la périphérie du centre ${ }^{10}$.

De par sa situation (ancienne «première »), on pourrait croire que la veuve est proche de «la première ». Pourtant, tout sépare Marie-Louise d'Aulnières de sa fille. Dès la naissance de cette dernière, la mère se détache du destin de son enfant et s'abîme dans le deuil de son époux :

Sa fille mise au monde, Mme d'Aulnières quitte le grand deuil pour entrer en demi-deuil, pour l'éternité. Costumée en grand-mère, malgré ses dixsept ans, robe noire, bonnet blanc, col et poignets de lingerie fine, elle entreprend de vieillir et de se désoler. $(K: 52)$

9. Tous les états de tierce se construisent en doublons : Florida/Aurélie, Mme d'Aulnières/Mme Tassy, les sœurs Lanouette/Léontine Mélançon. Les attributs physiques et psychologiques de chaque état sont reproduits sur deux générations : la servante/sorcière et la vieille fille reviennent, par des personnages différents, dans les deux temps de la narration du roman. Quant à l'état de veuve, il est représenté par deux personnages connexes, les mères des personnages principaux. L'état de femme semblerait donc façonner davantage une identité économico-sexuelle : en effet, il s'infiltre dans la psyché de chaque personnage.

10. Nous n'avons pas inclus le personnage de Horse Marine, la « femme de mauvaise vie », la « seconde femme » qu'Antoine fréquente régulièrement à Sorel. Cependant, elle n'est en rien une menace pour « la première » (Elisabeth réagit davantage avec fatalisme qu'avec colère ou déception face à cette liaison); de plus, la narration nous empêche d'accéder véritablement au personnage. Horse Marine est une ombre qui plane sur I'honneur de la famille, certes, pas sur l'héroïne. De même, nous ne traiterons pas du personnage de Sœur Catherine des Anges. 
Si Elisabeth fait rapidement la découverte de sa désirabilité et du plaisir de la chair, sa mère en revanche se retranche dans un monde asexué certain. Elle s'est même détournée entièrement du fruit de son union sexuelle avec le défunt $M$. d'Aulnières. Elle est exclue du champ d'action du roman, en marge de l'intrigue amoureuse et sexuelle dans laquelle sa fille joue le rôle principal. Elle n'agit qu'aux périphéries, approuvant malgré elle les réalisations charnelles d'Elisabeth. Elle « [s]e décide à parler » $(K: 60)$ en agréant le projet des sœurs Lanouette qui souhaitent trouver un mari pour Elisabeth. Elle dit « oui » au mariage de sa fille avec Antoine Tassy par convenance plus que par implication réelle ( $K: 68)$. De même, elle est présente lors des deux premières visites du docteur Nelson à Elisabeth, malade. Mme d'Aulnières n'est qu'une figure de légitimation de la sexualité, tant dans le mariage que dans l'adultère.

Il en va de même avec la seconde veuve du roman, Catherine des Rivières Tassy. Tout comme Marie-Louise, la mère d'Antoine évoque le stéréotype de la veuve recluse : «Son petit œil marron, rivé sur moi, ses grosses paupières plissées qui ne clignent pas. [...] Couleur de poussière, dressée de toute sa filiforme et frétillante petite personne. [...] Elle s'appuie sur une canne ». ( $K: 77-78)$ Elle rappelle Mme d'Aulnières par son comportement : «Pas plus qu'elle ne pleure, ma belle-mère ne rit jamais. » ( $K: 79)$ Aigrie, Mme Tassy garde un œil sur Elisabeth, mais surtout sur son propre fils, dont elle autorise les pratiques sexuelles conjugales - elle reste garante d'Antoine - et adultérines. Elle recommande à sa bru de ne pas accorder trop d'importance à la vie de débauche du jeune homme ( $K: 78)$. Dans le même temps, elle infantilise les activités libidinales de son fils : Iorsque la jeune fille partie en « voyage à Québec » $(K: 79)$ avec Antoine meurt, elle s'empresse de donner un conseil à «ce petit ». ( $K: 79)$ Aussi, sans être partie prenante d'une économie sexuelle directe, dénuées de tout attrait pouvant attiser les envies sexuelles, les deux veuves de Kamouraska agissent en appuyant et en légitimant les désirs charnels des personnages.

Similaire à l'état de veuve sur de nombreux points, l'état de vieille fille, « forcément ridicul[e] $\gg$ (Heinich, $1996: 257$ ), est aussi très présent dans le roman, avec Léontine Mélançon (qui a cette « odeur aigre de vierge mal lavée » $[K: 31]$ ) et surtout les tantes d'Elisabeth. «Étoffes noires, mates [...] bijoux d'améthyste et d'argent [...] [t]rois corps d'oiseaux momifiés dans leurs plumes ternies » $(K: 97)$ : les sœurs Lanouette renvoient aux clichés associés à la vieille fille dans la littérature occidentale. Pieuses et consciencieuses, elles mettent dans « la Petite » toutes 
les aspirations frustrées de leur existence de célibataire, dans un $19^{\text {e }}$ siècle où les « filles qui ne se marient pas », ont une position sociale marginale. Leur description devient franchement caricaturale lorsque la narratrice précise combien elles aiment les romans d'amour et que leur rapport au monde est « fabuleux et romanesque. » ( $K: 55)$ Les tantes Lanouette font la projection de leurs rêves hérités des œuvres sentimentales. De fait, bien qu'elles soient elles aussi à la périphérie de la sexualité, elles n'en sont pas moins agentes. Très souvent, les trois vieilles filles sont des complices : elles mentent lors du jugement d'Elisabeth ( $K: 45$ ), elles l'introduisent dans le système économico-sexuel (« Nous l'amènerons au bal du gouverneur. Nous lui ferons faire un grand mariage. »[K:47]) Parfois, la morale chrétienne reprend le dessus, et les «trois vieilles fées » mettent en garde leur nièce : « C'est un bien grand péché, ma petite fille. » ( $K: 161)$ Mais les sœurs Lanouette sont majoritairement des adjuvantes qui, sous des dehors involontaires, sont en réalité des spectatrices des désirs assouvis d'Elisabeth. Le paroxysme de ce double visage se confirme lors de la fuite de tante Adélaïde avec la jeune femme : par cet acte, la sœur Lanouette affiche une relative complaisance dans sa contemplation des péripéties charnelles d'Elisabeth. En outre, si elles apprécient de manière implicite le roman de la chair qui se joue devant leurs yeux, les trois sœurs n'en comprennent pas pour autant les rouages. Elles demeurent interdites devant les premières règles de la jeune fille ( $K: 55)$, tout comme elles sont saisies devant la saturation du rouge dans l'ouvrage d'Elisabeth $(K: 43)$.

Elisabeth attend une aide précise, celle d'Aurélie. Elle est la servante et la sorcière, celle qui exécute, mais aussi celle qui sait. Sa connaissance libidinale est théorique. Dans un premier temps, elle n'est pas agente, mais voyante : « Et pour ce qui est de la honte, autant vous habituer tout de suite. Cela ne fait que commencer. Le pire n'est pas encore arrivé. » ( $K: 105)$ Ni première ni seconde, Aurélie se situe aux confins de la tierce durant la majeure partie du roman, une tierce qui juge sa maîtresse («Votre histoire d'amour avec Monsieur le docteur me fait mourir, Madame ! » [ $K: 172])$, mais en subit les conséquences dans le même temps, puisqu'elle prend le crime passionnel sur elle. Le pouvoir d'agir d'Aurélie se déplace au moment où elle devient complice de l'assassinat, lors d'une soirée près du feu avec George et Elisabeth. Instant d'initiation, passation symbolique du meurtre des mains du couple à celles de la servante, cette veillée évoque les scènes rituelles sataniques: Aurélie est une sorcière, et le docteur le diable $(K: 175)$. Mais la scène est aussi imprégnée d'une sensualité, ne serait-ce que parce que le porto et le feu alanguissent les per- 
sonnages qui se serrent et s'appuient les uns contre les autres $(K: 178)$. Aurélie est déshabillée par les deux autres personnages : «Enlevons le corsage et la jupe. Nous nous passons le corps léger, de main en main. » $(K: 179)$ Le corps se montre, on touche les «épaules maigres » $(K: 179)$ et les «petits seins d'Aurélie. » $(K: 179)$ Pour que la servante tue le mari, il faut lui transmettre les pouvoirs divins d'un « saint » (c'est George qui se voit ainsi $[K: 179]$ ) ou d'un « diable » par un rapport sexuel sous-jacent qui coïncide avec les récits populaires autour du sabbat. Tout n'est que suggestion de l'acte, et la veillée au coin du feu s'achève sur une Aurélie qui a revêtu les vêtements d'Elisabeth. Aussi, la tierce se confond avec la première, le rite initiatique et sexué altère les rapports maître-valet conventionnels. Intégrée malgré elle au système libidinal, la servante s'affiche franchement comme sorcière, « une femme dans tous [s]es états ». (Heinich, $1996: 290)^{11}$

Dans Kamouraska, le système agentif des personnages est régi principalement par la sexualité d'un sujet, Elisabeth. De fait, l'absence de sexualité des autres femmes nous renseigne sur le champ des possibles dans la diégèse. La grille d'analyse de Heinich rend compte d'une diversité et en même temps d'une unité des identités de femmes dans le texte romanesque par le prisme de la relation sexuelle. Plus encore, elle renseigne sur l'investissement idéologique évident de la sexualité, terrain de bataille où des forces sociales tutélaires exploitent les acteurs et les corps dans le souci d'une vision du monde qui dépasse le désir strictement sexuel.

\section{Enjeux d'un assujettissement sexuel}

Dès le début du roman, Elisabeth prend la parole. Le « je » s'anime et analyse son quotidien d'épouse et de mère :

Épouse parfaite de Jérôme Rolland, un petit homme doux qui réclame son dû presque tous les soirs, avant de s'endormir [...]. Mon devoir conjugal sans manquer. Règles ou pas. Enceinte ou pas. Nourrice ou pas. Parfois même le plaisir amer. L’humiliation de ce plaisir volé à l'amour. $(K: 10)$

L'amour est dès le départ exclu du paysage romanesque. L'acte sexuel est un devoir accompli par les deux époux. Qu'importe la situation, c'est une routine. Cette répétition de tous les jours fait tomber la satisfaction des sens de I'héroïne dans une machinisation du corps féminin, un corps mis au service du mari dans le but de contenter ce dernier et de faire des enfants. Elisabeth est partie prenante du rituel économique

11. Force est de constater que les deux femmes de chambre, à savoir Aurélie et Florida, sont les seuls personnages féminins, exceptée Elisabeth, en contact physique avec des hommes - Antoine pour Aurélie, M. Rolland pour Florida : elles sont des « femmes à la bouteille », celles qui administrent le poison ou l'antidote, des sorcières. 
du coït décidé par le mari12. L'incipit s'ouvre sur une femme passive et consciente de cette même passivité, dont l'acte sexuel est un marqueur. En même temps, Elisabeth exprime un premier désir autonome, celui d'être vue par les autres hommes en même temps qu'elle les voit, dans un échange de regards qui apparaît comme un privilège recouvert : «Relever mon voile de deuil. Regarder tous les hommes, dans la rue. Tous. Un par un. Être regardée par eux. » $(K: 9)$ Le simple regard échangé entre la femme et les hommes connote un plaisir non dissimulé, le premier signe d'une attirance sexuelle à fleur de peau. Dès lors, Elisabeth se dédouble : sous les traits de la femme rangée, contrainte à la monotonie d'un acte sexuel impersonnel et commun, se cache un être de chair fièrement désirant. En témoigne ce «plaisir amer $(K: 10)$ qui l'extirpe parfois de sa torpeur, faiblesse sensible révélatrice d'un désir sexuel exacerbé. La sexualité est dès le départ au service de la collectivité : il faut produire des enfants. Le sujet féminin semble être en décalage par rapport à cette logique, et déjà le désir de subversion apparaît. Cela, Jérôme Rolland I'a bien compris. Lorsqu'Elisabeth se prend à rêver - et donc à s'échapper, ne serait-ce qu'en pensées, de sa condition - il intervient de manière indirecte dans la narration : « Il faudrait avoir la santé de violer cette femme. La ramener de force avec nous, sur le lit conjugal. [...] L'obliger à penser à nous, à souffrir avec nous, à partager notre agonie, à mourir avec nous. » $(K: 26)$ Le rapport sexuel est aussi un rappel à l'ordre du mari, une sanction pour la femme mariée qui a voulu s'échapper un instant de sa position dans le système patriarcal, soit celle d'un objet soumis à l'autorité du mari.

Dans Kamouraska, le sexe féminin est un lieu de bataille et de conquête. La relation entre Elisabeth et Antoine commence dans un décor de chasse, où I'un et l'autre sont à la fois prédateurs et proies. Plus tard, par une alternance des voix narratives, le « je » masculin de George évoque son désir de « posséder cette femme » $(K: 129)$, de la conquérir, comme une terre appartenant à Antoine : «Couchée dans le lit d'Antoine, battue par Antoine, caressée par Antoine, ouverte et refermée par Antoine, violée par Antoine, ravie par Antoine. » $(K: 129)$ Ce n'est pas un simple « combat de coqs » qui a lieu entre deux hommes, mais bien la rupture d'un contrat social instable entre deux puissances idéologiques et culturelles qui s'affrontent au-dessus du corps féminin. D'un côté, il y a Antoine Tassy, le blond : il est le seigneur de Kamouraska, Canadien français, catholique, héritier d'un attachement à la terre et aux traditions féodales dont il est le modèle et le garant; sa vie constitue le centre des sociabilités

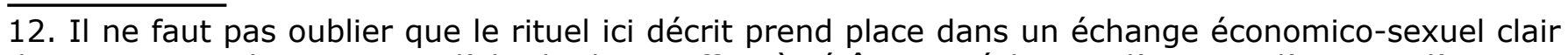
dans Kamouraska. Le corps d'Elisabeth est offert à Jérôme en échange d'un toit, d'argent, d'un nom social et surtout d'une protection légale qui légitime la présence la jeune femme dans la société après sa condamnation. 
rurales. De l'autre côté, le noir George Nelson est l'Américain, le marginal, qui n'habite pas Sorel, mais la campagne environnante. Protestant, il a renié sa foi. Il est l'étranger, l'Autre, un imposteur dont on se méfie bien qu'il soit médecin - peut-être parce qu'il a droit de vie et de mort sur les autres.

Les commentateurs ont vu qu'avec Kamouraska, Anne Hébert réinventait I'histoire canadienne-française du $19^{\mathrm{e}}$ siècle tout en l'extrayant du passé pour l'interpréter à la lumière des questionnements des années 1960 et 1970, notamment avec la ré-

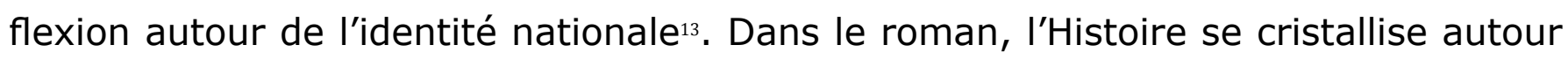
d'un sexe, celui du personnage féminin, lieu de pouvoir et de contestation. Tour à tour, Elisabeth est prise par Antoine, George, puis Jérôme Rolland, dans un coït qui matérialise à la fois la domination de la terre, mais aussi la loi du Père sur l'objet-femme ${ }^{14}$. Mais I'héroïne ne peut supporter cette supériorité du sexe masculin sur le sien. Aussi, le refus du coït est hautement significatif. Une première fois, elle se soustrait à l'agonie de son mari Jérôme, qui ne peut la violer et la ramener dans le «droit chemin ». Pour se donner à George, figure de l'Autre et d'un contre-pouvoir, elle se refait une virginité en refusant son lit à Antoine : «intouchée, intouchable, sauf pour l'unique homme de ce monde, en marche vers moi. » ( $K: 117)$ Mais dans le même temps, nous avons vu que l'acte sexuel était un pacte entre les époux, tout comme il est une entente tacite entre Jérôme et Elisabeth. Espace de conquête, mais aussi de négociation et de médiation, vectrice et fonction des rapports de force entre personnages, ainsi qu'entre plusieurs enjeux sociétaux, la sexualité est envisagée par I'héroïne comme un terrain propice à l'assujettissement, c'est-à-dire à l'assimilation de sa domination par un autre et à la compréhension de sa subjectivité ${ }^{15}$. Dès lors, l'assujettissement est vécu doublement par Elisabeth et son partenaire sexuel, dans un échange agentif de pouvoir.

$\overline{13 . ~ «[C] ' e s t ~ q u e ~ K a m o u r a s k a ~ n ' e s t ~ p a s ~ u n e ~ f i c t i o n ~ q u i ~ s e ~ l o g e ~ d a n s ~ I ' H i s t o i r e, ~ j u d i c i a i r e ~ o u ~ a u t r e, ~ e l l e ~}$ est une fiction qui redonne à l'Histoire son vécu avec le regard de la contemporaine qu'elle ne cesse jamais d'être » (Beaulieu, 1970 : 50) ; « Même pour le lecteur québécois, ce livre constituera un dépaysement total. Il ne connaît d'habitude rien de son histoire et Anne Hébert a écrit un roman qui a comme trame cette histoire elle-même. [...] Elle y fait revivre le passé et c'est tout naturellement le présent qui prend forme, qui pleure, qui tue, qui va et vient, qui se perd et se retrouve et nous avec lui dans une sorte de merveilleuse aspiration. » (Éthier-Blais, 1970 : 12) On lira aussi la critique de Réginald Martel parue dans La Presse le 10 octobre 1970.

14. Nous nous référons ici au schéma de la structure patriarcale de Patricia Smart dans Écrire dans la maison du père (1988).

15. Assujettissement au sens foucaldien et tel qu'il est repris par Judith Butler dans La vie psychique du pouvoir : « En tant que forme de pouvoir, I'assujettissement est paradoxal. La domination par un pouvoir extérieur à soi est une forme bien connue, douloureuse, prise par le pouvoir. Découvrir, néanmoins, que ce que I"on" est, sa propre formation de sujet, dépend en un sens de ce même pouvoir en est une autre [...]. Le pouvoir n'est pas simplement ce à quoi nous nous opposons, mais aussi, en un sens fort, ce dont dépend notre existence, ce que nous abritons et conservons dans les êtres que nous sommes. » (Butler, 2002 : 23) 
Refus ou soumission au coït, tractation ou routine, ou encore accomplissement d'un fantasme : quoi qu'il en soit, parce qu'elle permet à Elisabeth de se considérer comme sujet, la sexualité subvertit des valeurs sociales et morales portées durant le $19^{e}$ siècle canadien-français. La relation avec le docteur Nelson est un désaveu face à Antoine et aux idées qu'il véhicule. Mais plus encore, il faut voir dans la sexualité la déviance par rapport à un discours étouffant hérité du jansénisme et de ses concepts de prédestination divine. Tous les personnages sont mauvais : Elisabeth est damnée, Antoine est rongé par « cette chose intolérable qui l'avilit à la racine même de sa vie » $(K: 146)$, George est le diable. Et tous cherchent à réprimer ce mal intérieur, les instincts les plus bas. Il y a un combat opposant la nature physique et impulsive, celle des sens et de la sensualité, et les impératifs sociaux et culturels, l'éducation et la religion qui façonnent les identités au profit d'une autre nature - fausse, celle$\mathrm{ci}$ - saine et respectueuse des codes. Le personnage principal rend compte de cette dichotomie : «Mon âme n'a pas encore rejoint mon corps. » $(K: 10)$ L'éducation reçue a évité d'aborder la question du corps et de ses envies, en construisant autour de l'amour un dispositif discursif porteur des valeurs chrétiennes. En utilisant les mots « chair » et « sexe » de manière récurrente, en mettant en scène les souvenirs des événements de sa jeunesse, Elisabeth s'arroge le droit de recréer un monde à son image : passionné et sensuel. Elle tente de rejeter la prescription et va à l'encontre des conventions - pour mieux réintégrer le discours prescriptif à la fin de son parcours, en se remariant et en revêtant le masque de l'innocence. En outre, selon Gabrielle Pascal-Smith, «Elisabeth revit éternellement son drame dans un présent infernal qui ne lui laisse aucun répit mais dont elle fait son ultime liberté. » (Pascal-Smith, 1980 : 90) Le souvenir de la sexualité est la possibilité de dépasser la condition de femme mariée soumise à des contraintes évidentes.

De la confusion des sentiments à la liberté conditionnelle d'Elisabeth, on comprend que le désir sexuel s'insinue dans les recoins du tissu romanesque construit par Anne Hébert. La notion de sexualité ne se réduit pas à l'acte sexuel, bien que ce dernier soit au coeur du système agentif du roman. Elle régit à la fois la trajectoire de I'héroïne, l'agencement des personnages et les rapports de force entre idéologies. Aussi, si les mots « chair » et « sexe » peuvent paraître tabous au $19^{e}$ siècle, ils n'en demeurent pas moins essentiels dans la construction et la perpétuation des tensions sociales à travers des actions rituelles ou marchandes, comme le mariage. Ceux qui ne sont pas agents de l'économie sexuelle demeurent en retrait du champ de pouvoir, 
forcés d'aider, de manière indirecte, le système à continuer - ou à se désintégrer. A contrario, Elisabeth, voyante et agente, «femme de théâtre » $(K: 78)$ et chair incorruptible, centralise les désirs des autres dans le système agentif et s'appuie sur eux pour parvenir à ses fins. De là naît l'intrigue de Kamouraska, c'est-à-dire lorsque le sujet intègre et réinterprète la sexualité comme une arme au service de sa propre conception du monde, quitte à en subir des conséquences néfastes pour lui. 


\section{Bibliographie}

BEAULIEU, Ivanhoé (1970), « Deux œuvres totalement différentes », Le Soleil, 26 septembre : 50.

BERNIER, Jovette-Alice (1931), La chair décevante, Montréal, Éditions Albert Lévesque, coll. "Les romans de la jeune génération $»$.

BUTLER, Judith (2002 [1997]), La vie psychique du pouvoir. L'assujettissement en théories, trad. Brice Matthieussent, Paris, Éditions Léo Scheer.

DIDIER, Béatrice (1991), L'écriture-femme, Paris, Presses universitaires de France, $3^{e}$ édition.

ÉTHIER-BLAIS, Jean (1970), « Kamouraska d'Anne Hébert : à lire avec les yeux de l'âme », Le Devoir, 19 septembre : 12 .

HAVERCROFT, Barbara (1999), « Quand écrire c'est agir : stratégies narratives d'agentivité féministe dans Journal pour mémoire de France Théoret », Dalhousie French Studies, nº 47 : 93-113.

HÉBERT, Anne (1970), Kamouraska, Paris, Seuil.

HÉBERT, Anne (2013), CEuvres complètes II. Romans (1958-1970) : Les chambres de bois, édition établie par Luc Bonenfant, suivi de Kamouraska, édition établie par Anne Ancrenat et Daniel Marcheix, Montréal, Les Presses de l'Université de Montréal, coll. « Bibliothèque du Nouveau Monde ».

HEINICH, Nathalie (1996), États de femme. L'identité féminine dans la fiction occidentale, Paris, Gallimard, coll. « NRF essais ».

HEINICH, Nathalie (2003), Les ambivalences de l'émancipation féminine, Paris, Albin Michel, coll. « Bibliothèque des idées $»$.

JARDIN, Claudine (1970), « Du sang sur la neige du Canada », Le Figaro, 16 novembre : 25.

LE GRAND, Albert (1971), « Kamouraska ou l'Ange et la Bête », Études françaises, vol. 7, n 2 : 119-143.

MARTEL, Réginald (1970), « Deux lectures de Kamouraska », La Presse, 10 octobre : D-3.

MATTEI, Claude (1970), « Cette année-là à Kamouraska... », Le Provençal/Dimanche, 11 octobre.

PASCAL-SMITH, Pascale (1980), « La condition féminine dans Kamouraska d'Anne Hébert », The French Review, vol. 54, n 1 , octobre : 85-92.

ROY, Camille (1931), « La chair décevante », Enseignement secondaire au Canada, 17e année, vol. 2, novembre : 97-99.

SMART, Patricia (1988), Écrire dans la maison du père. L'émergence du féminin dans la tradition littéraire du Québec, Montréal, Québec/Amérique.

SMITH, Donald (1980-1981), « Anne Hébert et les eaux troubles de l'imaginaire », Lettres québécoises, $\mathrm{n}^{\circ} 20: 64-73$.

TABET, Paola (2004), La grande arnaque. Sexualité des femmes et échange économico-sexuel, trad. Josée Contréras, Paris, L'Harmattan.

THÉRIO, Adrien (1971), « La maison de la belle et du prince ou l'enfer dans l'œuvre romanesque d'Anne Hébert », dans Livres et auteurs québécois 1971, Montréal, Éditions Jumonville : 274-284.

VIGNEAUX, Jean (1971), « Kamouraska Anne Hébert », Pourquoi pas ?, 21 janvier : 100-105.

ZWEIG, Stefan (1992 [1927]), La confusion des sentiments, Paris, Livre de poche. 